\title{
Development of a backward-mode photoacoustic microscope using a Fabry-Pérot sensor
}

Pohle, Ulrike, Baumann, Elisabeth, Pulwer, Silvio, Villringer, Claus, Zhang, Edward, et al.

Ulrike Pohle, Elisabeth Baumann, Silvio Pulwer, Claus Villringer, Edward Zhang, Holger Gerhardt, Jan Laufer, "Development of a backward-mode photoacoustic microscope using a Fabry-Pérot sensor," Proc. SPIE 10878, Photons Plus Ultrasound: Imaging and Sensing 2019, 108786L (6 March 2019); doi: $10.1117 / 12.2525785$

SPIE. Event: SPIE BiOS, 2019, San Francisco, California, United States 


\title{
Development of a backward-mode photoacoustic microscope using a Fabry-Pérot sensor
}

\author{
Ulrike Pohle ${ }^{a, f, *}$, Elisabeth Baumann ${ }^{b, \dagger}$, Silvio Pulwer ${ }^{c}$, Claus Villringer ${ }^{a, c}$, Edward Zhang ${ }^{d}$, \\ Holger Gerhardt ${ }^{\mathrm{b}}$, Jan Laufer ${ }^{\mathrm{a}}$ \\ ${ }^{a}$ Institut für Physik, Martin-Luther-Universität Halle-Wittenberg, Von-Danckelmann-Platz 3, 06120 \\ Halle (Saale), Germany \\ ${ }^{\mathrm{b}}$ Max-Delbrück-Center for Molecular Medicine in the Helmholtz Association, Robert-Roessle-Str. \\ 10, 13092 Berlin, Germany \\ ${ }^{\mathrm{c}}$ Technische Hochschule Wildau, Hochschulring 1, 15745 Wildau, Germany \\ ${ }^{\mathrm{d}}$ Department of Medical Physics and Biomedical Engineering, University College London, Gower \\ Street, London WC1E 6BT, United Kingdom \\ ${ }^{\dagger}$ Contributed equally to this work \\ * Correspondence to ulrike.pohle@physik.uni-halle.de
}

\begin{abstract}
Optical-resolution photoacoustic microscopy (PAM) has been shown to enable the acquisition of high resolution ( $\mu \mathrm{m})$ functional and anatomical images. For backward-mode operation, conventional piezoelectric ultrasound transducers need to be placed far away from the signal source due to their opacity and size. This can result in reduced acoustic sensitivity. Planar Fabry-Perot polymer film interferometer (FPI) sensors have the potential to overcome this limitation since they are transparent to the excitation wavelength, can be placed immediately adjacent to the signal source for high acoustic sensitivity, and offer a broadband frequency response $(0-50 \mathrm{MHz})$.

In this study, we present a high frame rate, backward-mode OR-PAM system based on a planar FPI ultrasound sensor. A ns-pulsed laser provides excitation pulses ( $<200 \mathrm{~nJ}$, maximum pulse repetition frequency $=200 \mathrm{kHz}, 532 \mathrm{~nm})$ to generate photoacoustic waves that are detected using a planar FPI sensor interrogated at $765-781 \mathrm{~nm}$. For backwardmode operation and highest acoustic sensitivity, the excitation and interrogation beams are coaxially aligned and rasterscanned. The optical transfer function of the sensor, the spatial resolution and the detection sensitivity were determined to characterise the set-up. Images of a leaf phantom and first in vivo images of zebrafish larvae were acquired.
\end{abstract}

This approach will enable fast 3D OR-PAM with high resolution and high sensitivity for functional and molecular imaging applications. FPI-based ultrasound detection also has the potential to enable dual-mode optical- and acousticresolution PAM and the integration of photoacoustic imaging with purely optical modalities such as multi-photon microscopy.

\section{INTRODUCTION}

Optical-resolution photoacoustic microscopy (PAM) is a technique for the acquisition of anatomical and functional information with resolution in the $\mu \mathrm{m}$-regime and a penetration depth of up to $1 \mathrm{~mm}$ [1]. Short (ns) excitation laser pulses are focussed into the target to generate photoacoustic (PA) waves, and the generated pressure field is detected using an ultrasound sensor. The excitation beam is then raster-scanned and the resulting PA data set is converted into a 3D image by converting time to depth using the speed of sound in tissue.

For many OR-PAM applications it is desirable to excite and detect the PA signal on the same side of the sample, which is referred to as backward-mode imaging. Using conventional and often bulky piezoelectric transducers for this detection

Photons Plus Ultrasound: Imaging and Sensing 2019, edited by Alexander A. Oraevsky, Lihong V. Wang,

Proc. of SPIE Vol. 10878, 108786L · @ 2019 SPIE · CCC code: 1605-7422/19/\$18 · doi: 10.1117/12.2525785 
mode can result in large source-detector distances and hence limited acoustic sensitivity. Fabry-Pérot interferometer (FPI) sensors can overcome this limitation as they are transparent to the wavelength of the excitation pulse. The sensor can be placed immediately adjacent to the PA signal source, thus minimizing the source-detector distance for maximum acoustic sensitivity. FPI sensors also exhibit a large and broadband frequency response [2,3], which is advantageous for high resolution imaging. Lastly, backward-mode detection using FPI sensors also offers the potential for multi-modal imaging in a single instrument, such as a combination with confocal or multiphoton optical microscopy techniques.

In this study, we present a backward-mode Fabry-Pérot-based scanner aimed at OR-PAM of vascularisation. The planar FPI sensor is characterised and PAM images of a leaf skeleton phantom are acquired. Initial, single-wavelength in vivo imaging of zebra fish larvae is demonstrated.

\section{METHODS}

The setup of the PAM is shown in figure 1. For excitation of the sample, short ns-pulses of high-repetition rate lasers in the visible wavelength region are used $(532 \mathrm{~nm}$ and $580-600 \mathrm{~nm})$. The output of a dye laser $(580-600 \mathrm{~nm})$ is guided through a spatial filter to improve the beam profile. A small fraction of the excitation light is directed to a photodiode to monitor the pulse energy and for triggering. A tuneable $\mathrm{cw}$ diode laser is used to interrogate the FPI sensor at wavelengths between $765 \mathrm{~nm}$ and $781 \mathrm{~nm}$. The excitation and interrogation beams are co-aligned and raster-scanned coaxially across the FPI sensor over an area of up to an $8 \times 8 \mathrm{~mm}^{2}$ using galvanometer mirrors. An objective lens $(\mathrm{f}=50 \mathrm{~mm})$ is used to focus the excitation beam into the sample while the interrogation beam is focused onto the FPI sensor at a distance of $1 \mathrm{~mm}$ below the excitation focal plane using additional lenses in the interrogation beam path.

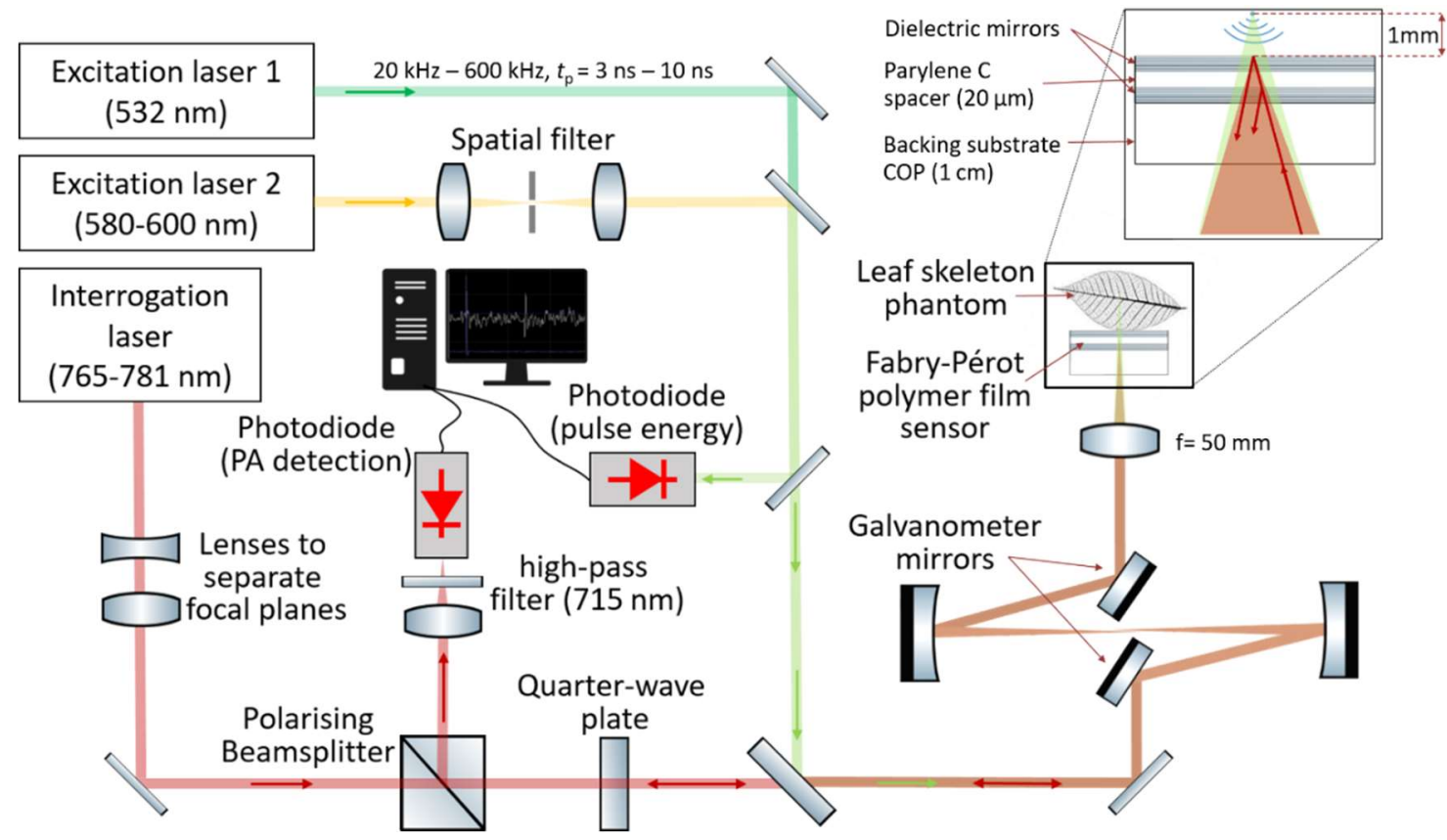

Figure 1: Schematic of the Fabry-Pérot optical-resolution PA microscope.

The PA waves generated by the excitation beam induce modulations of the optical thickness of the FPI sensor by altering the thickness of the parylene spacer. Thus a phase shift between the interrogation beam reflected from the two dielectric mirrors of the interferometer occurs, which was detected as a change in the reflected optical power using a Siphotodiode. A $715 \mathrm{~nm}$-cut-off filter is used to prevent the detection of scattered excitation light. 
For the imaging experiments, excitation pulses of $10 \mathrm{~ns}$ at a wavelength of $532 \mathrm{~nm}$ and a $20 \mathrm{kHz}$ pulse repetition frequency were used. For the in vivo measurements, zebra-fish larvae (4 days post fertilization, strain AB/TÜLF) were anesthetised with tricaine (3-amino benzoic acidethylester) and immobilised in agarose gel.

\section{RESULTS}

\subsection{Optical and acoustic properties of the FPI sensor}

The mirrors of the FPI sensor were designed to transmit the excitation laser wavelength, whereas the interrogation laser wavelengths are reflected $(\mathrm{R}=98 \%$ at $790 \mathrm{~nm})$. The resulting transmission spectrum of a single mirror is shown in figure 2(A).

The acoustic frequency response of a sensor with soft dielectric mirrors and a $20 \mu \mathrm{m}$ parylene spacer was simulated using a k-wave program [5] and is displayed in figure 2(B). The estimated bandwidth of this sensor is $55 \mathrm{MHz}(-3 \mathrm{~dB}$ level).
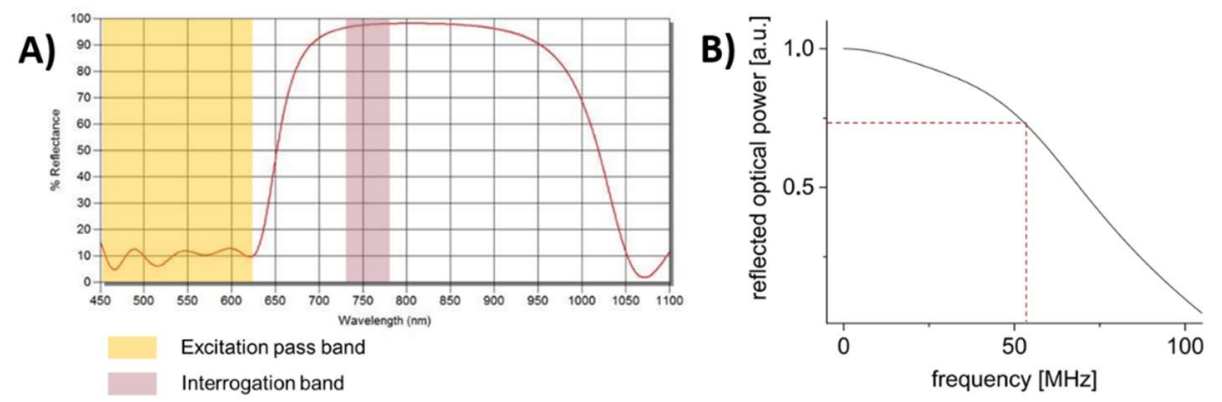

Figure 2: Characterisation of FPI sensor (A) Transmission spectrum of the dielectric mirrors, (B) Simulated acoustic frequency response.

\subsection{PA microscopy of a leaf skeleton}

An image of a leaf skeleton phantom was acquired at an excitation wavelength of $532 \mathrm{~nm}$, using a pulse energy of $125 \mathrm{~nJ}$ and a step size of $10 \mu \mathrm{m}$. The maximum intensity projections (MIP) of the 3D image data set are shown in figure 3.


Figure 3: Maximum intensity projections of a leaf skeleton. (A) z plane, (B) x plane, (C) y plane. 


\subsection{In vivo PA microscopy of zebra fish larvae}

In an initial study, in vivo images of wild-type zebra fish larvae were obtained at a pulse energy of 200nJ. In figure 4(A) a MIP acquired with a step size of $10 \mu \mathrm{m}$ is shown. For comparison, a bright-field microscopic image with anatomical annotations is displayed in figure 4(B).

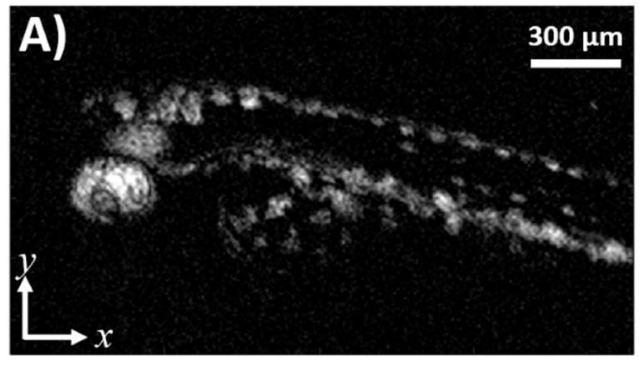

B)

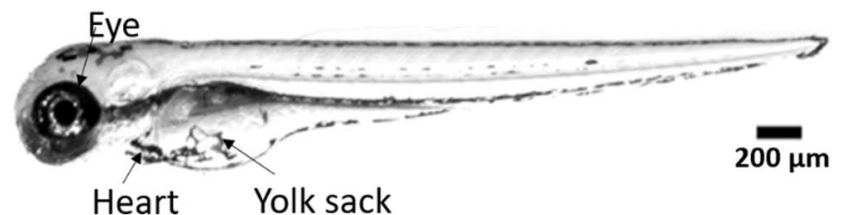

Figure 4: Images of a zebra fish larvae (A) MIP acquired with the PA microscope, (B) bright-field microscopic image of a zebra fish larvae.

\subsection{Spatial resolution}

The lateral resolution was obtained from measurements of the edge-spread function of a 2D MIP image of gold foil. An image intensity profile perpendicular to the edge was taken, as indicated by the white line in figure 5(A). A Boltzmann function was fitted to the edge-spread function, which is displayed in figure 5(B). To estimate the lateral resolution, the first derivative of the Boltzmann function was fitted with a Gauss function, as shown in figure 5(C), and the FWHM was obtained. This procedure was repeated in five different locations, resulting in a mean lateral resolution of $8.5 \mu \mathrm{m} \pm 4.3 \mu \mathrm{m}$. The highest resolution was $6.5 \mu \mathrm{m}$. The axial resolution is limited by the excitation pulse length of $10 \mathrm{~ns}$ and was estimated as $15 \mu \mathrm{m}$.
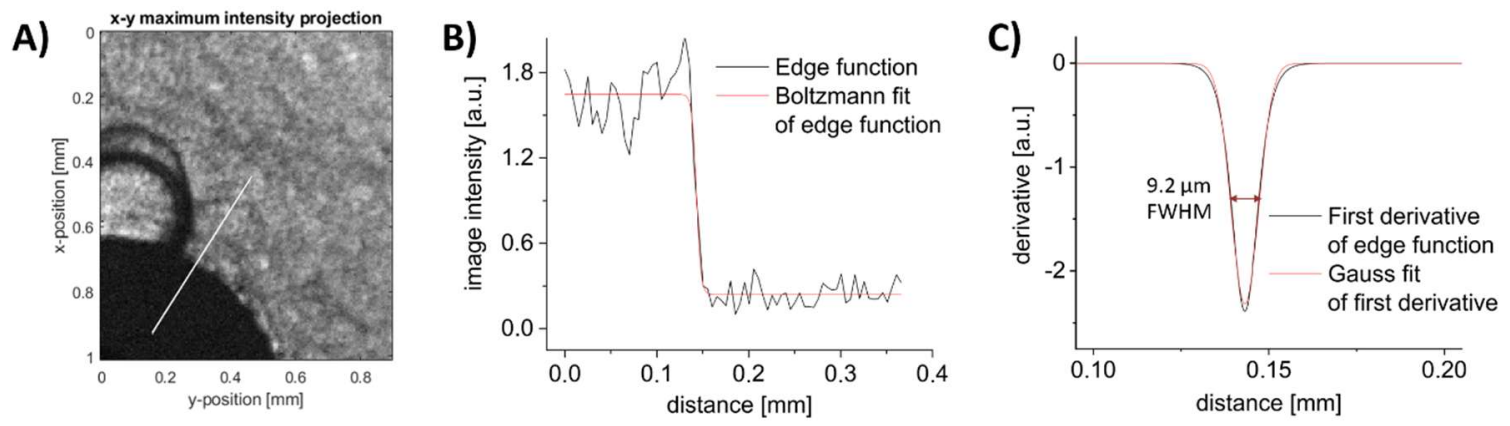

Figure 5: Measurement of the lateral resolution (A) 2D MIP image of gold foil, (B) Image intensity profile corresponding to the solid white line shown in (A) and a fitted Boltzmann function, (C) First derivative of the fitted Boltzmann function in (B) and a Gaussian fit for comparison.

\section{CONCLUSION}

In this work, a backward-mode FPI-based optical-resolution PA microscope was demonstrated. The system combines broad detection bandwidth $(55 \mathrm{MHz}$ at $-3 \mathrm{~dB})$ and with a lateral and axial resolution of $8.5 \mu \mathrm{m}$ and $15 \mu \mathrm{m}$, respectively. Initial phantom and in vivo images indicate potential for pre-clinical research. 


\section{ACKNOWLEDGEMENTS}

This work was funded by Deutsche Forschungsgemeinschaft (DFG, LA 3273/5-1, GE 2154/1-1).

\section{REFERENCES}

[1] Yao, J. and Wang, L.V. “Photoacoustic microscopy”, Laser Photonics Rev. 7(5), 758-778 (2013).

[2] Buchmann et al. "Characterization and modeling of Fabry-Perot ultrasound sensors with hard dielectric mirrors for photoacoustic imaging", Appl. Opt. 56(17), 5039-5046 (2017).

[3] Zhang, E., Laufer, J. and Beard, P.C. "Backward-mode multiwavelength photoacoustic scanner using a planar Fabry-Perot polymer film ultrasound sensor for high-resolution three-dimensional imaging of biological tissue" Appl. Opt. 47, 561-577 (2008).

[4] Liu et al. "Quad-mode functional and molecular photoacoustic microscopy" Sci. Rep. 8, 2018.

[5] Beard, P.C., Perennes, F. and Mills, T.N. "Transduction mechanisms of the Fabry-Perot polymer film sensing concept for wideband ultrasound detection" IEEE Trans. Ultrason. Ferroelect. Freq. Control 46 (6), 1575-1582 (1999).

[6] Zhang et al. "A Naturally-Derived Compound Schisandrin B Enhanced Light Sensation in the pde6c Zebrafish Model of Retinal Degeneration" PLOS One, 2016. 